\title{
The effect of different bromine sources on the dual cation mixed halides perovskite solar cells (Supporting Information)
}

\author{
Damian Głowienka ${ }^{1,2},{ }^{*}$ Francesco Di Giacomo ${ }^{2,3}$, Mehrdad Najafi ${ }^{2}$, Ilker Dogan ${ }^{2}$, \\ Alfredo Mameli ${ }^{4}$, Fallon J. M. Colberts ${ }^{5}$, Jędrzej Szmytkowski ${ }^{1}$, and Yulia Galagan ${ }^{2,6 \dagger}$ \\ ${ }^{1}$ Faculty of Applied Physics and Mathematics, Gdańsk University of Technology, Narutowicza 11/12, 80-233 Gdańsk, Poland \\ 2 TNO - Solliance, High Tech Campus 21, Eindhoven 5656AE, The Netherlands \\ ${ }^{3}$ Centre for Hybrid and Organic Solar Energy (CHOSE), Department of Electronic Engineering, \\ University of Rome Tor Vergata, Via del Politecnico 1, 00133 Rome, Italy \\ ${ }^{4}$ TNO - Holst Centre, High Tech Campus 31, Eindhoven 5656AE, The Netherlands \\ ${ }^{5}$ Molecular Materials and Nanosystems, Institute for Complex Molecular Systems, \\ Eindhoven University of Technology, P.O. Box 513, Eindhoven 5600 MB, The Netherlands \\ ${ }^{6}$ Department of Materials Science and Engineering, \\ National Taiwan University, Taipei 10617, Taiwan
}

In the drift-diffusion model, the potential distributions is calculated based on the Poisson equation which links all the charge carriers densities together

$$
\frac{\partial^{2} \phi}{\partial x^{2}}=-\frac{q}{\varepsilon_{0} \varepsilon_{r}}\left(p-n+N_{D}-N_{A}\right)
$$

where $\phi$ represents a local electric potential which depends on the material permittivity $\left(\varepsilon_{r}\right)$ and charge carrier concentrations. Here, the charge is possesed by free electrons $(n)$ and holes $(p)$, and also by the concentration of p-type doping $\left(N_{A}\right)$ and the n-type doping $\left(N_{D}\right)$. The constants $\varepsilon_{0}$ and $q$ represent a vacuum permittivity and an elementary charge, respectively. The electric field is in the following relation with a potential distribution

$$
F=-\frac{\partial \phi}{\partial x}
$$

The transport of charge carriers is calculated using the continuity equations for electrons and holes [1]

$$
\begin{aligned}
& \frac{\partial n}{\partial t}=G-R_{m}-R_{b}-R_{t}+\frac{1}{q} \frac{\partial}{\partial x}\left[-q n \mu_{n} \frac{\partial \phi}{\partial x}+\mu_{n} k_{B} T \frac{\partial n}{\partial x}\right], \\
& \frac{\partial p}{\partial t}=G-R_{m}-R_{b}-R_{t}-\frac{1}{q} \frac{\partial}{\partial x}\left[-q p \mu_{p} \frac{\partial \phi}{\partial x}-\mu_{p} k_{B} T \frac{\partial p}{\partial x}\right],
\end{aligned}
$$

where $T$ represents absolute temperature and $k_{B}$ is the Boltzmann constant. $\mu_{n(p)}$ is the mobility of electrons (holes) and $J_{n}$ and $J_{p}$ represent electron and hole current densities, respectively. The charge carriers are generated with a given generation rate $(G)$ taken from the generation profile, and also recombine with a monomolecular recombination rate $\left(R_{m}\right)$, a bimolecular recombination rate $\left(R_{b}\right)$ and a trimolecular recombination rate $\left(R_{t}\right)$. The total current is calculated for each position in space averaged throughout the whole simulated solar cell

$$
J_{t o t}(x)=J_{n}(x)+J_{p}(x)+J_{d i s p}(x) .
$$

The time dependent electric field results in displacement current density which is defined as

$$
J_{d i s p}=\varepsilon_{r} \varepsilon_{0} \frac{\partial F}{\partial t} .
$$

\footnotetext{
* damian.glowienka@pg.edu.pl

† yulia.galagan@tno.nl
} 
The generation model is based on the transfer matrix model $[2,3]$. The optical parameters $\eta$ and $\kappa$ for the calculations are used the same as given in the previous work of our group [4]. The monomolecular recombination is defined with the SRH model. Usually, this model assumes the trap location in the middle of the band-gap where the recombination is the most efficient [5]. However, it is not always possible to use a simple model with only one recombination center. In closer to reality scenario, the traps are distributed with different traps energies. Therefore, the density of states (DOS) is commonly applied to explain the traps distribution [6, 7]. The trap-assisted recombination model is then calculated by summing all the trapped electrons from the conduction band (CBT)

$$
R_{m, C B T}(x)=\int_{E_{\nu}}^{E_{c}} \rho_{T}(E) R_{S R H, n}(E, x) d E
$$

and holes from the valence band (VBT)

$$
R_{m, V B T}(x)=\int_{E_{\nu}}^{E_{c}} \rho_{T}(E) R_{S R H, p}(E, x) d E,
$$

where $\rho_{T}$ is the DOS for traps, and $R_{S R H, n(p)}$ are recombination rates for electrons

$$
R_{S R H, n}(E, x)=\frac{C_{n c} C_{p c} N_{t n}}{C_{n c}\left[n(x)+n_{1}(E)\right]+C_{p c}\left[p(x)+p_{1}(E)\right]}\left[n(x) p(x)-n_{i n t}^{2}\right]
$$

and holes

$$
R_{S R H, p}(E, x)=\frac{C_{n \nu} C_{p \nu} N_{t p}}{C_{n \nu}\left[n(x)+n_{1}(E)\right]+C_{p \nu}\left[p(x)+p_{1}(E)\right]}\left[n(x) p(x)-n_{i n t}^{2}\right],
$$

where, $C_{n c}$ and $C_{p c}$ are trapping and detrapping rates for electrons from the conduction band, respectively, and $C_{p \nu}$ and $C_{n \nu}$ represent trapping and detrapping rates for holes from the valence band, respectively. The detrapping processes associated with $C_{p c}$ and $C_{n \nu}$ are related with capturing of opposite charge carriers. Parameters $N_{t n}$ and $N_{t p}$ represent trap states densities for electrons and holes, respectively. The total recombination can be defined as the sum of both rates $R_{m}(x)=R_{m, C B T}(x)+R_{m, V B T}(x)$. The energy (E) dependent parameters $n_{1}$ and $p_{1}$ are given by

$$
\begin{gathered}
n_{1}(E)=N_{c} \exp \left(-\frac{E_{c}-E_{t}}{k_{B} T}\right), \\
p_{1}(E)=N_{\nu} \exp \left(-\frac{E_{t}-E_{\nu}}{k_{B} T}\right), \\
n_{\text {int }}=\left(N_{c} N_{\nu}\right)^{1 / 2} \exp \left(-\frac{E_{g}}{2 k_{B} T}\right),
\end{gathered}
$$

where $N_{c}$ and $N_{\nu}$ are effective densities of states for conduction and valence bands, respectively. Also, $E_{c}$ and $E_{\nu}$ denote energy edges of conduction and valence bands, respectively. $E_{t}$ is a trap energy level which is located in the given energy location. The DOS function is usually characterized by an exponential density function for shallow traps close to the energy band $[8,9]$ and a Gaussian density function for deep traps [10]. However, it has been shown that the exponential trap distribution can be also simulated with the Gaussian function [7]. Therefore in this work, $\rho_{T}$ is only defined with a Gaussian distribution

$$
\rho_{T}(E)=\frac{N_{t}}{\sqrt{2 \pi \sigma_{t}}} \exp \left[-\frac{\left[E-\left(E_{c}-E_{t}\right)^{2}\right]}{2 \sigma_{t}^{2}}\right],
$$

where $\sigma_{t}$ is the width of the distribution. The bimolecular recombination of charge carrier density is based on the Langevin model, while the trimolecular recombination is defined with the Auger recombination mechanism [1]. 
The injection barrier at the interfaces of HTL/perovskite and perovskite/ETL are simulated with the generalized potential. The method includes energy variations at the interfaces with the modified Poisson equation [11-13]

The time boundary conditions at initial time $(t=0)$ are all fixed to zero values. Also, the Schottky contacts are assumed at both metal contacts. We may distinguish positions at the bottom anode $(x=0)$ electrode

$$
n(0)=N_{c} \exp \left(\frac{-\phi_{n}}{k_{B} T}\right), p(0)=N_{v} \exp \left(\frac{\phi_{n}-E_{g}}{k_{B} T}\right),
$$

and at the top cathode $(x=L)$ from the other side

$$
n(L)=N_{c} \exp \left(\frac{\phi_{p}-E_{g}}{k_{B} T}\right), p(L)=N_{v} \exp \left(\frac{-\phi_{p}}{k_{B} T}\right),
$$

where $\phi_{n}$ and $\phi_{p}$ denote work functions for the both aforementioned electrodes. The electric potential at the boundaries are also given by

$$
\phi(0)=V_{\text {built }}-V_{a}, \phi(L)=0 .
$$

The built-in voltage $\left(\mathrm{V}_{\text {built }}\right)$ and the applied voltage $\mathrm{V}_{a}$ are fixed values in the model. The convergence condition for numerical calculations is defined as

$$
\left|\frac{J_{\text {total }}^{t+1}-J_{\text {total }}^{t}}{J_{\text {total }}^{t}}\right| \leqslant 10^{-9} .
$$

The discretization of all equations has been accomplished with the Scharfetter-Gummel [14] and the finite difference schemes. The more detailed equations are shown in Głowienka et al. [4].

\section{RESULTS}


(a)

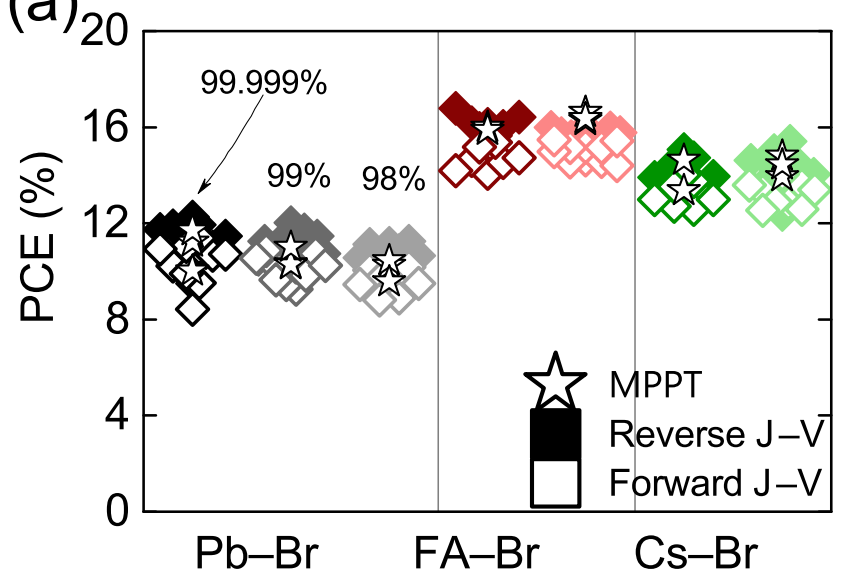

(c)

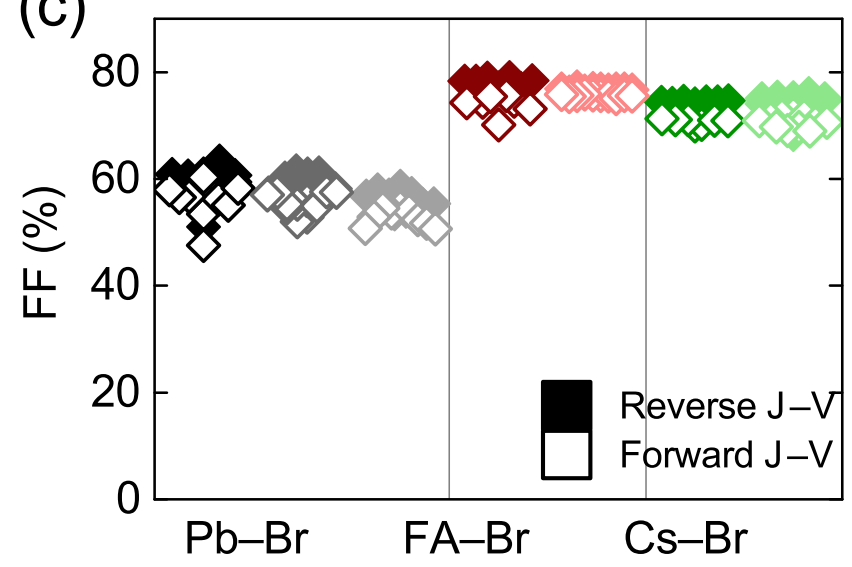

(b)

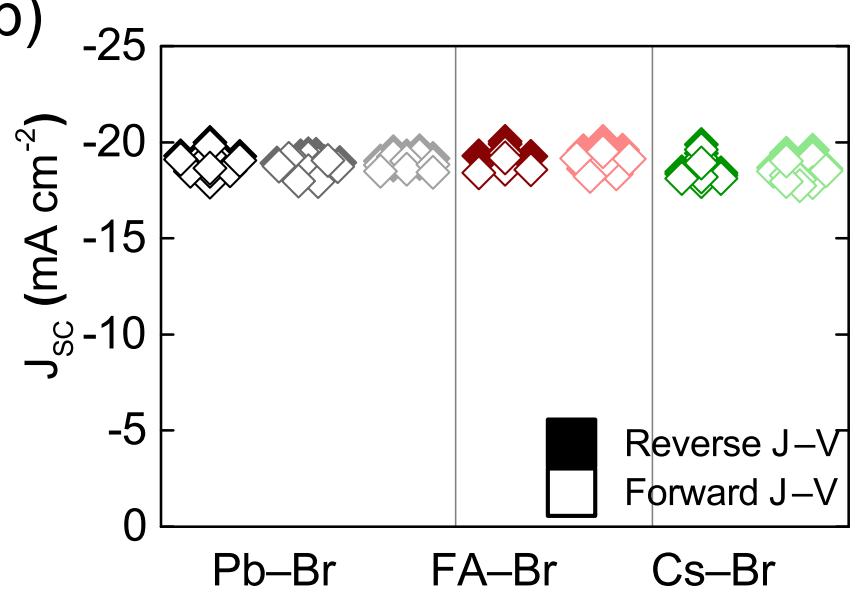

(d)

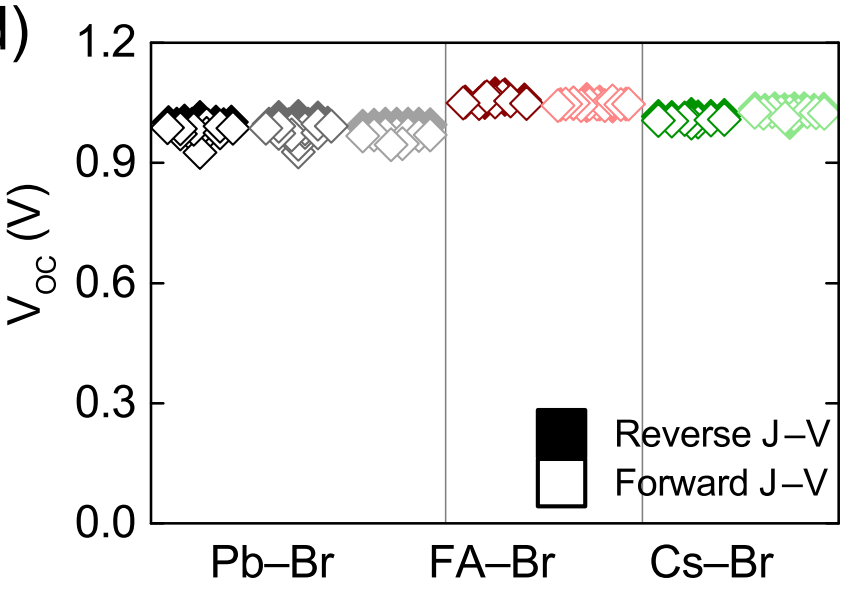

FIG. S1. Photovoltaic parameters for PSCs, a) PCE, b) $\mathrm{J}_{s c}$, c) FF, and d) $\mathrm{V}_{o c}$. Solar cells with the different bromide source and the alternative supplier to exclude possible impurities effect. The perovskite with $\mathrm{Pb}-\mathrm{Br}$ (black points), FA-Br (red points) and $\mathrm{Cs}-\mathrm{Br}$ (green points) source of bromide. The darker colors correspond to regular supplier and the lighter in respect to new supplier with different purity of the compound 
(a)

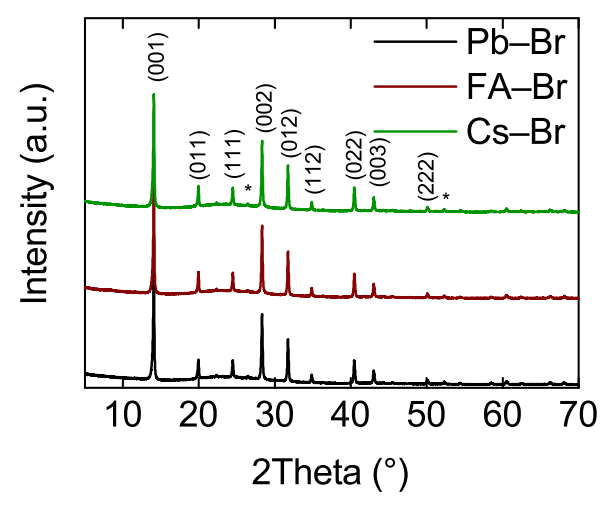

(b)

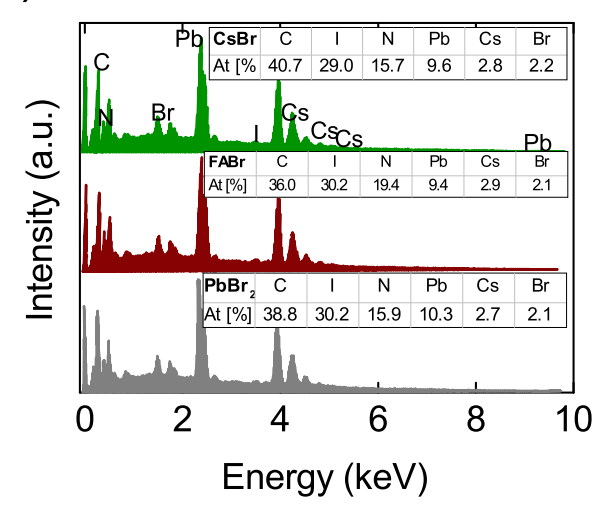

(c)

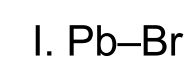

II. $\mathrm{FA}-\mathrm{Br}$

III. Cs-Br
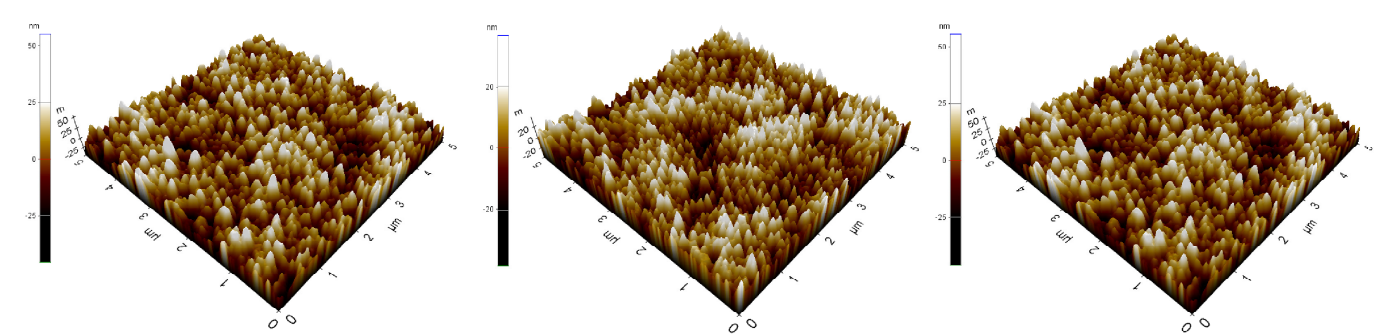

(d)
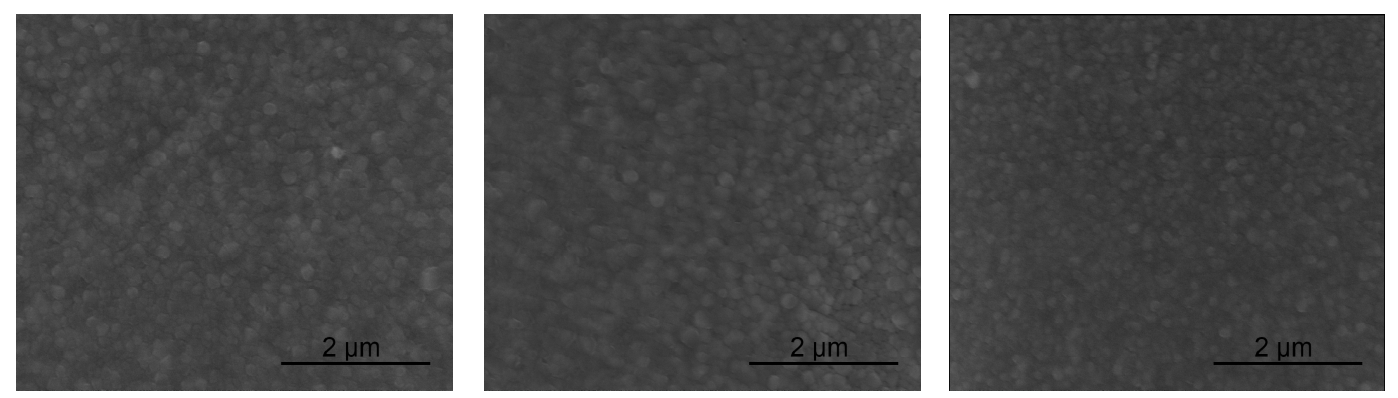

FIG. S2. Experimental results for the perovskite layer on glass. a) XRD, b) EDX, c) AFM, and d) SEM measurements. The perovskite with $\mathrm{Pb}-\mathrm{Br}$ (black line), $\mathrm{FA}-\mathrm{Br}$ (red line) and $\mathrm{Cs}-\mathrm{Br}$ (green line) source of bromide. 

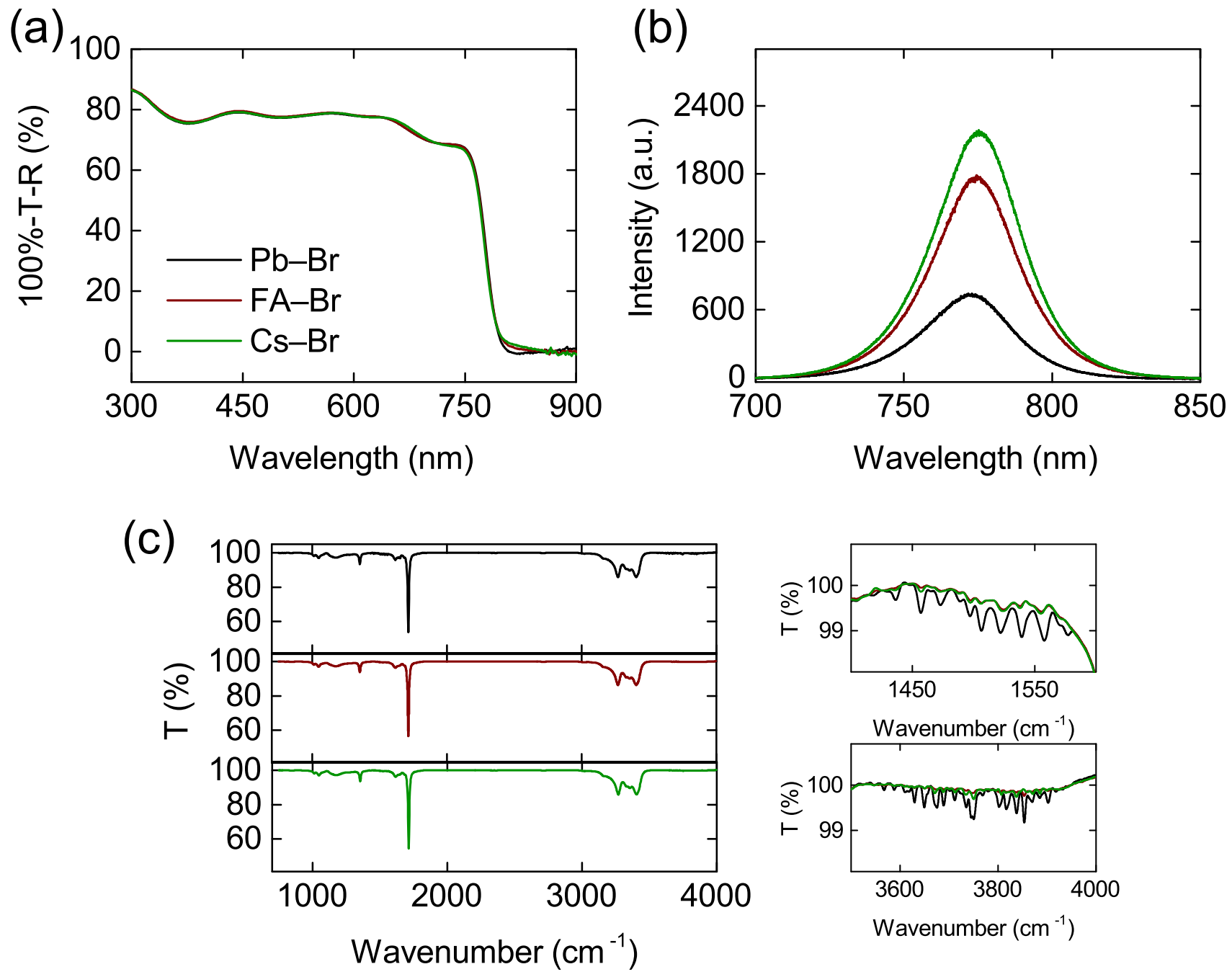

FIG. S3. Experimental results for the perovskite layer. a) UV-vis, b) PL and c) FTIR measurements with two insets. The perovskite with $\mathrm{Pb}-\mathrm{Br}$ (black line), $\mathrm{FA}-\mathrm{Br}$ (red line) and $\mathrm{Cs}-\mathrm{Br}$ (green line) source of bromide. The perovskite layers are prepared on the glass (for a and b) and on the silicon (for c) substrates. 

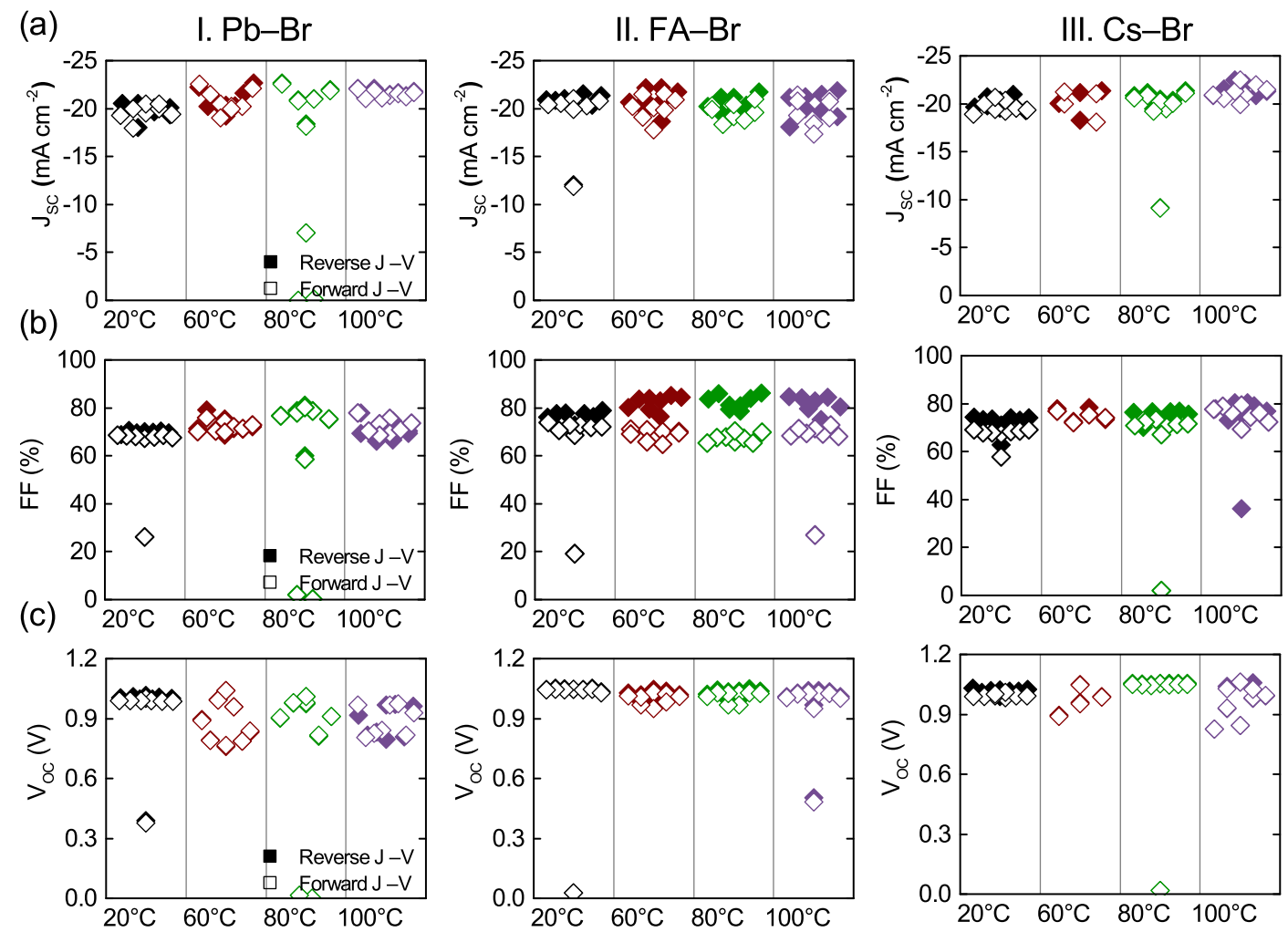

FIG. S4. Photovoltaic parameters for PSCs, a) $\mathrm{J}_{s c}$, b) FF, and c) $\mathrm{V}_{o c}$. Solar cells with heating of the solution overnight in $20^{\circ} \mathrm{C}$ (black points), $60^{\circ} \mathrm{C}$ (red points), $80^{\circ} \mathrm{C}$ (green points) and $100^{\circ} \mathrm{C}$ (violet points). The perovskite with $\mathrm{Pb}-\mathrm{Br}$ (section $\mathrm{I}$ ), $\mathrm{FA}-\mathrm{Br}$ (section II) and $\mathrm{Cs}-\mathrm{Br}$ (section III) source of bromide.

(a)

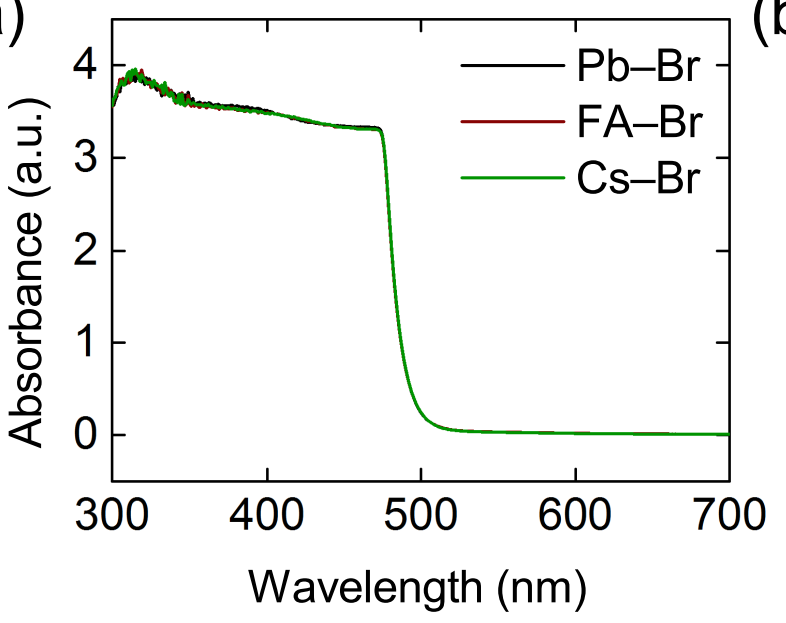

(b)

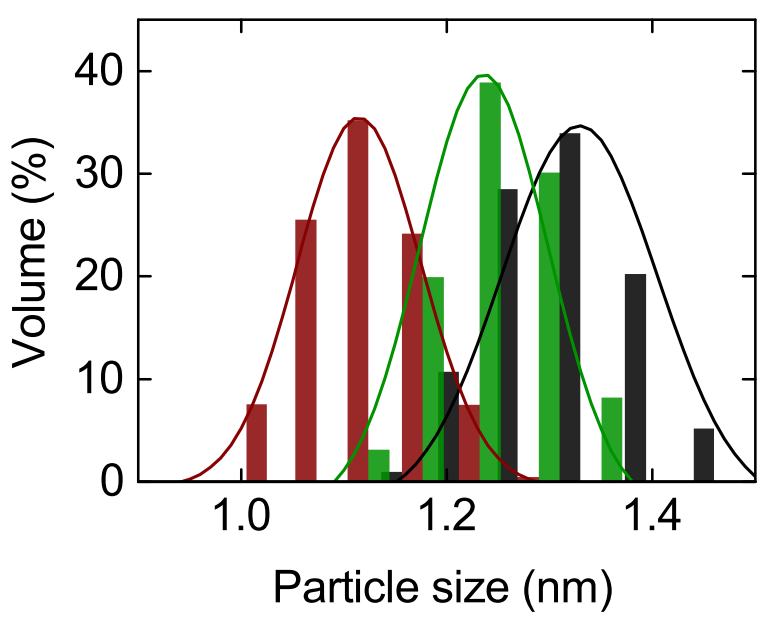

FIG. S5. The experimental results for three perovskite solutions. a) UV-vis, b) DLS measurements. Black, red and green colors represent perovskite solutions with $\mathrm{Pb}-\mathrm{Br}, \mathrm{FA}-\mathrm{Br}$ and $\mathrm{Cs}-\mathrm{Br}$ bromide source, respectively. 

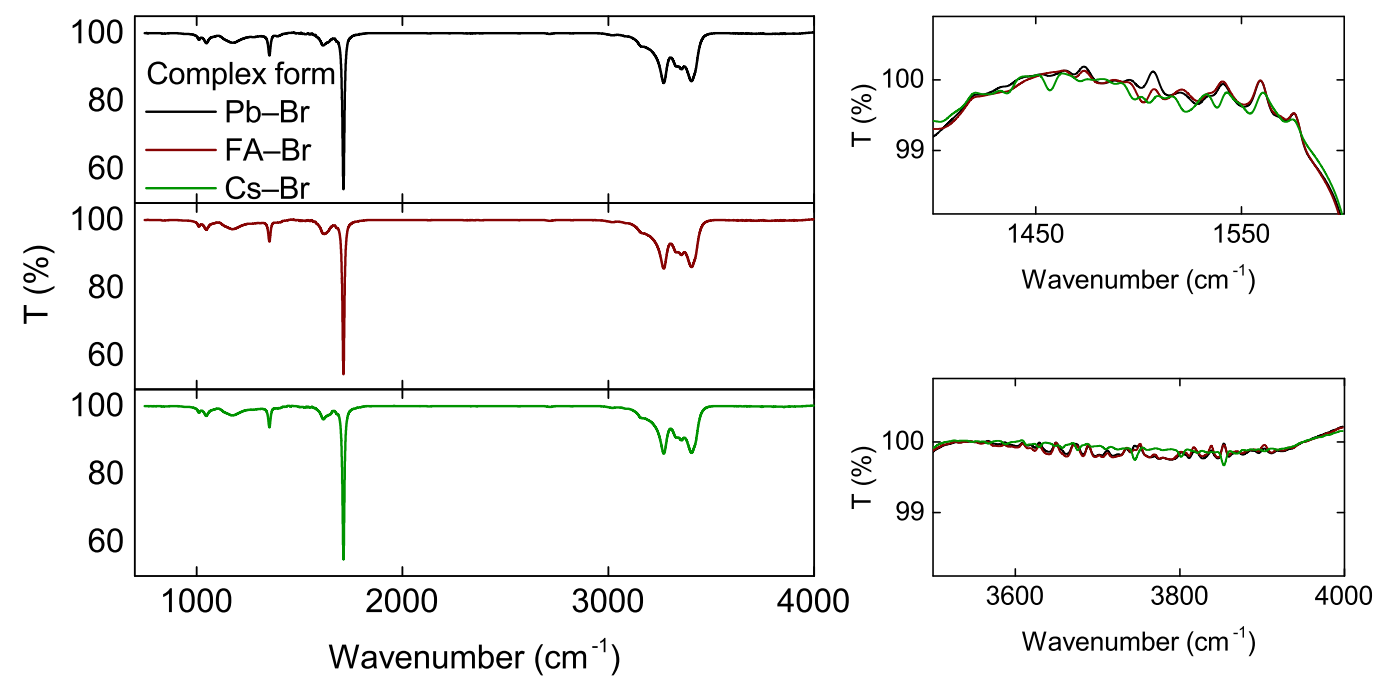

FIG. S6. FTIR results for not-annealed perovskite layer on the silicon substrate with two insets. The perovskite with $\mathrm{Pb}-\mathrm{Br}$ (black line), $\mathrm{FA}-\mathrm{Br}$ (red line) and $\mathrm{Cs}-\mathrm{Br}$ (green line) source of bromide.

(a)

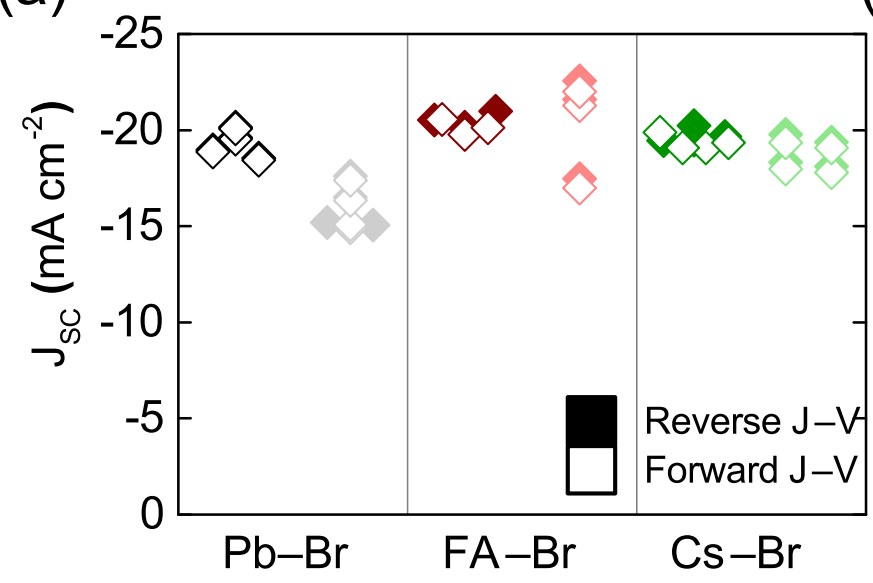

(b)

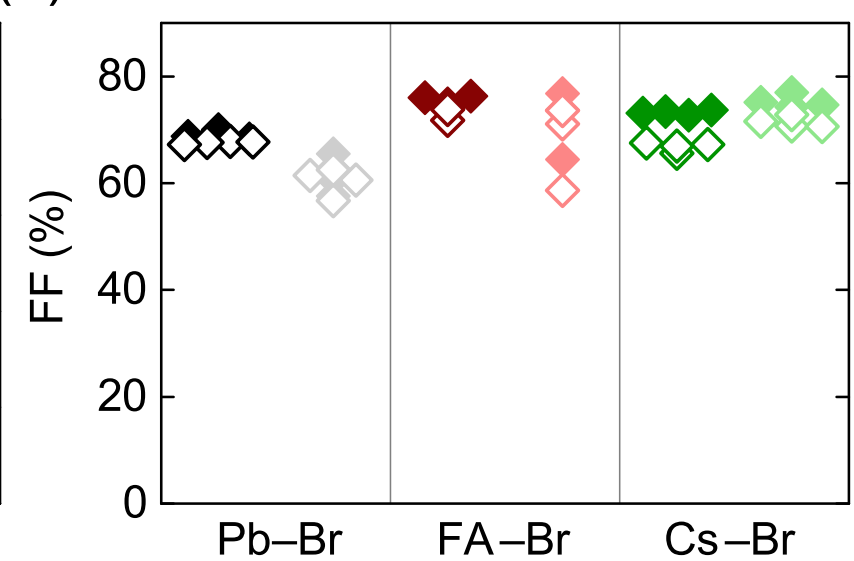

(c)

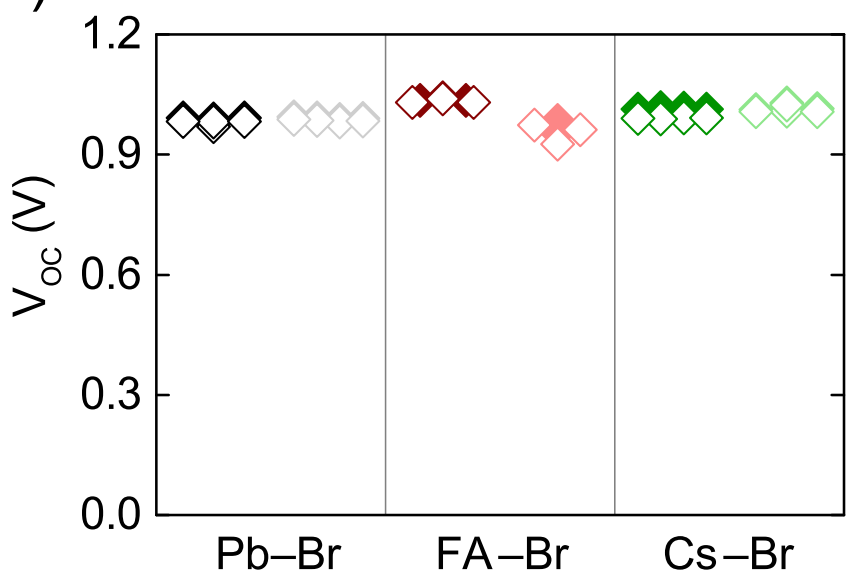

FIG. S7. Photovoltaic parameters for PSCs, a) $\mathrm{J}_{s c}$, b) FF, and c) $\mathrm{V}_{o c}$. Solar cells with the different bromide source and the degradation effect after 9 months. The perovskite with $\mathrm{Pb}-\mathrm{Br}$ (black points), $\mathrm{FA}-\mathrm{Br}$ (red points) and Cs-Br (green points) source of bromide. The darker colors correspond to fresh samples and the lighter in respect to 9 months old cells. 
(a)

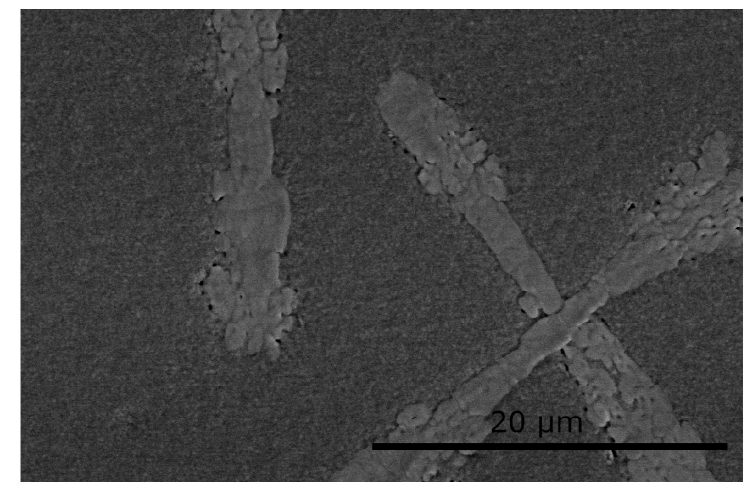

(c)

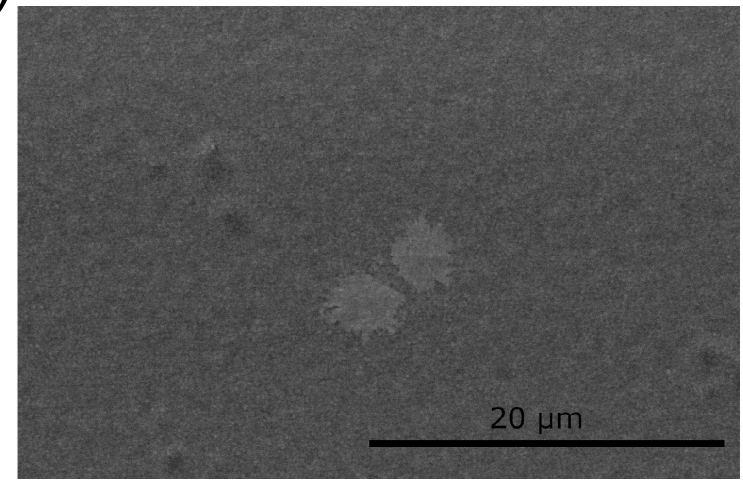

(b)

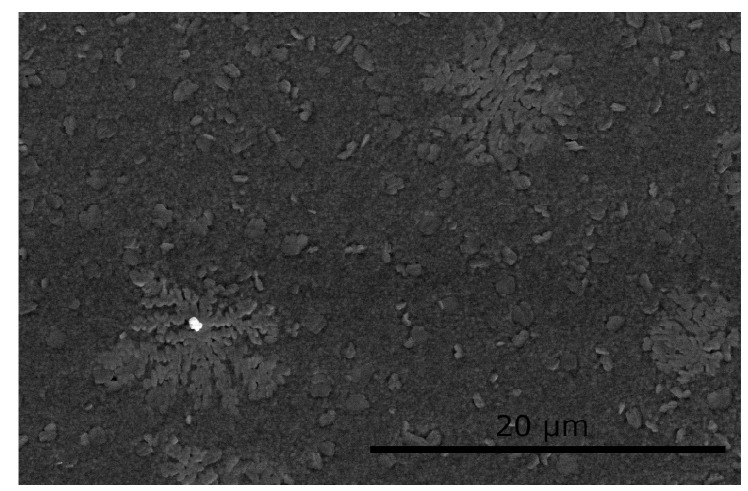

(d)

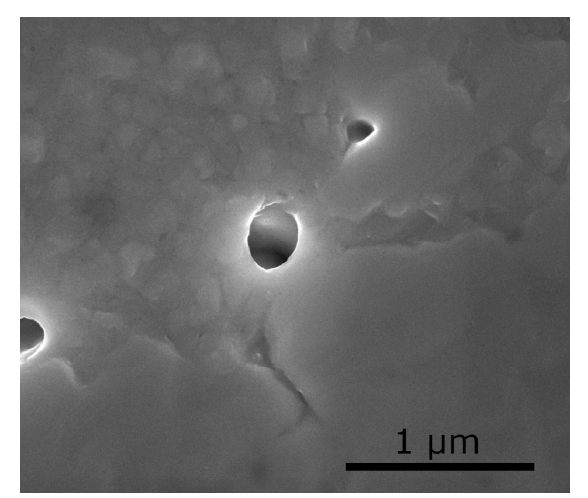

FIG. S8. SEM results for the perovskite layer on glass after 9 months storage. The perovskite with a) $\mathrm{Pb}-\mathrm{Br}$, b) FA-Br, c) $\mathrm{Cs}-\mathrm{Br}$ source of bromide, and d) $\mathrm{Pb}-\mathrm{Br}$ with borders of two crystals. 
(a)

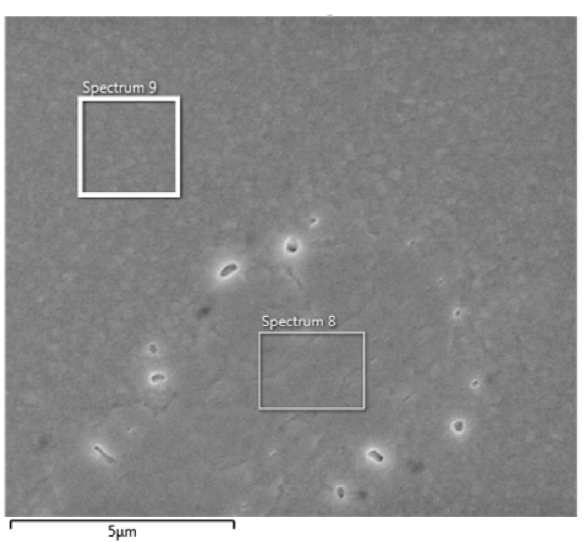

(b)

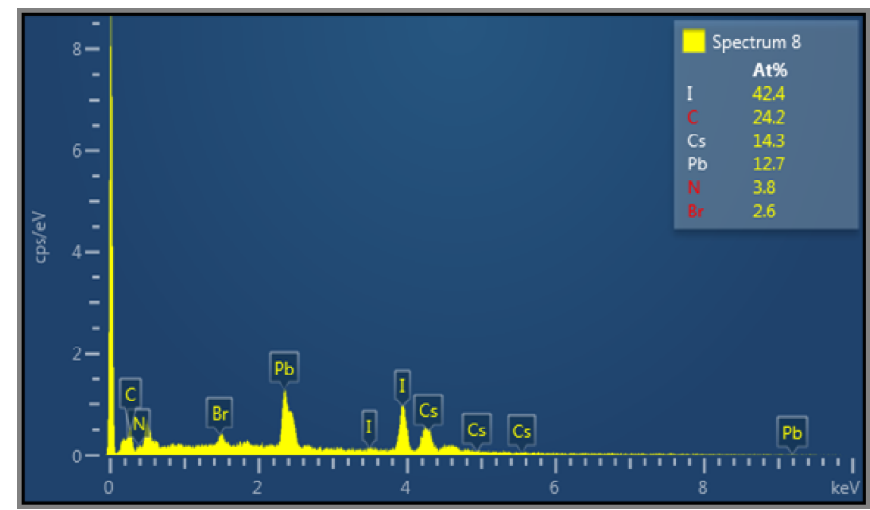

(c)

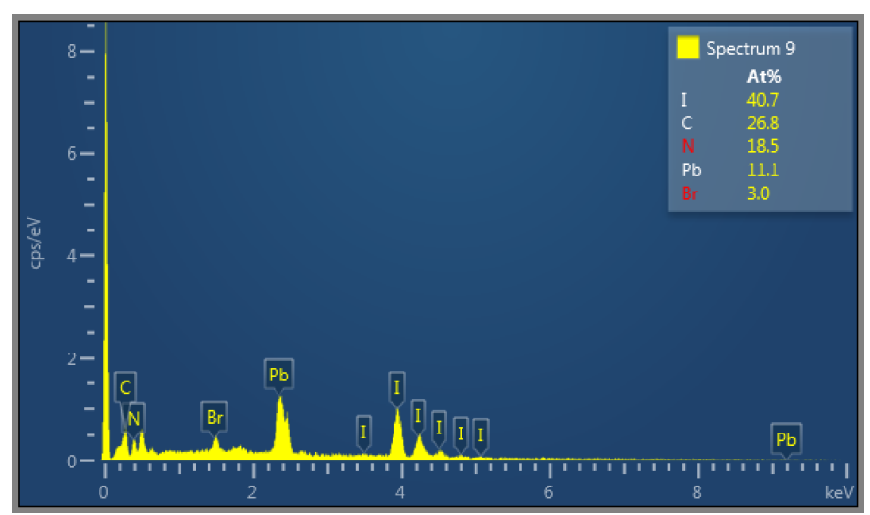

FIG. S9. EDX results for the $\mathrm{Pb}-\mathrm{Br}$ perovskite layer on glass after 9 months of storage. a) SEM image pointing the place of EDX spectrum measurements ( $b$ and $c)$. 
(a)

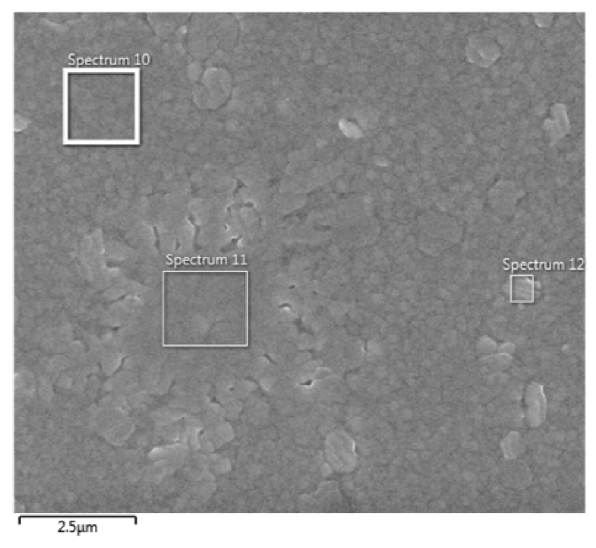

(b)

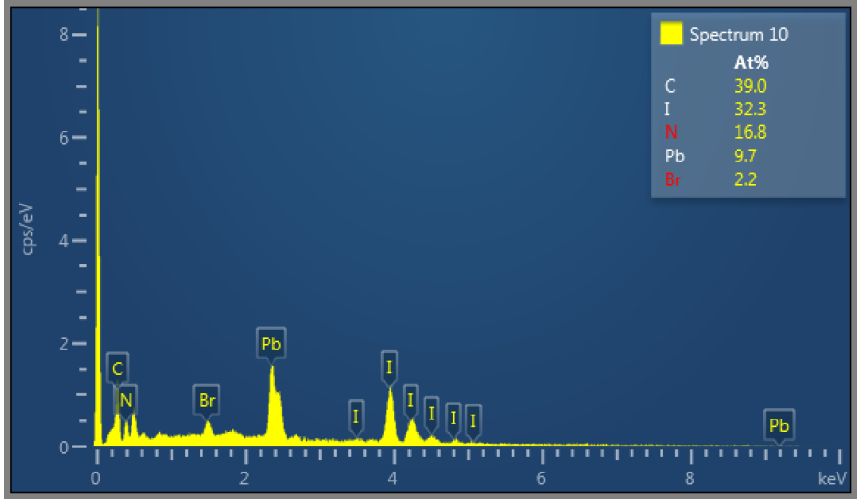

(c)

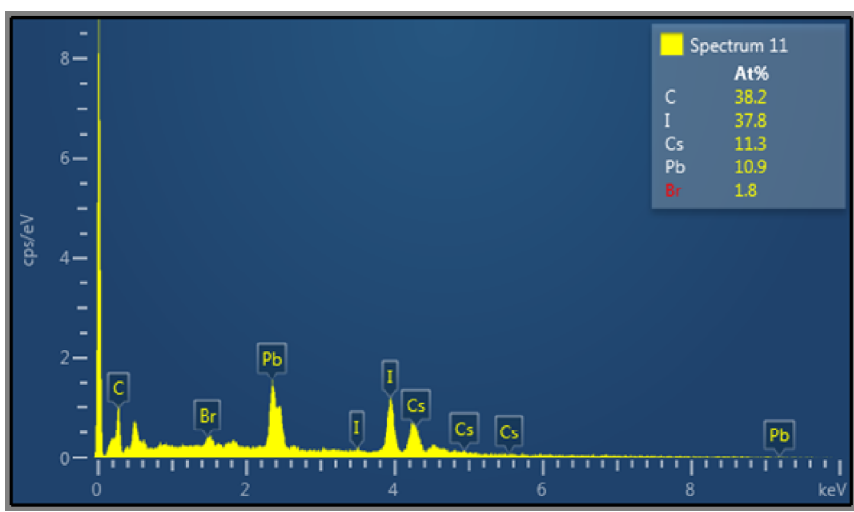

(d)

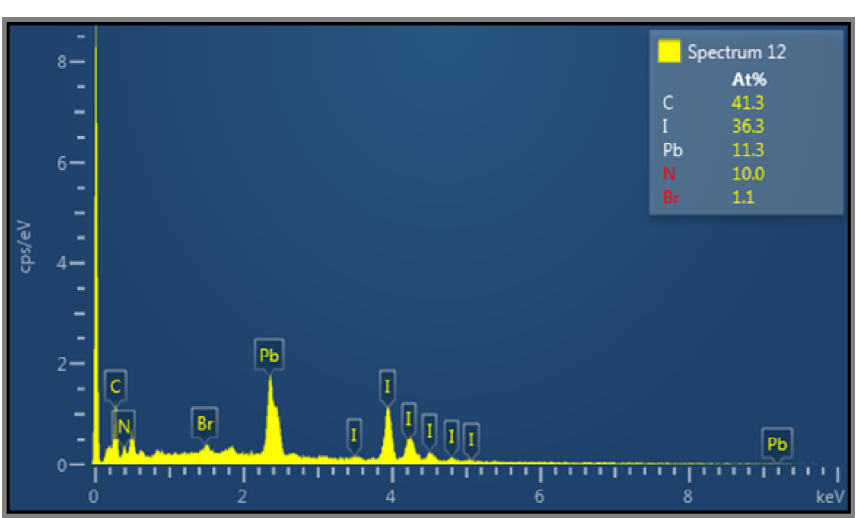

FIG. S10. EDX results for the FA-Br perovskite layer on glass after 9 months of storage. a) SEM image pointing the place of EDX spectrum measurements (b-d). 
(a)

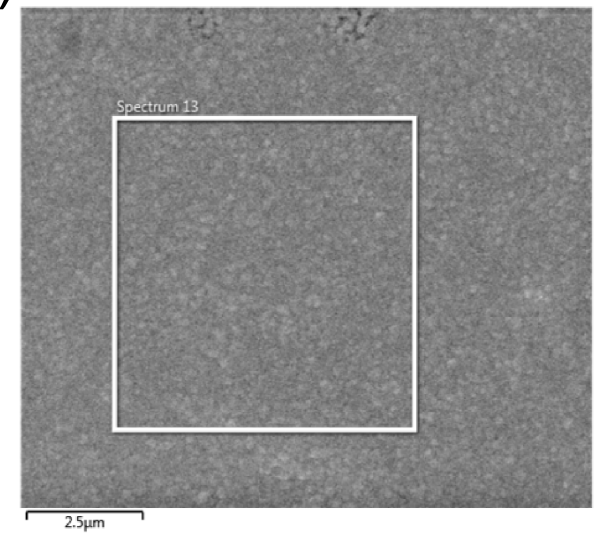

(b)

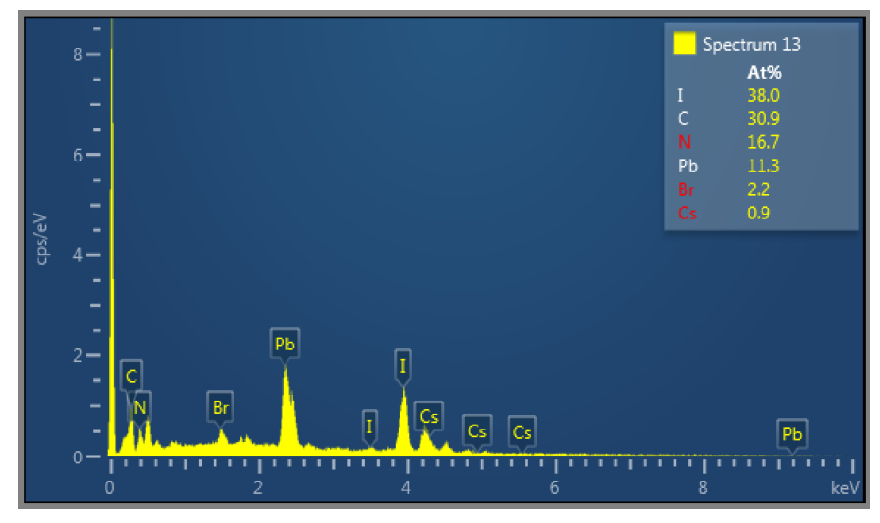

FIG. S11. EDX results for the Cs-Br perovskite layer on glass after 9 months of storage. a) SEM image pointing the place of EDX spectrum measurements (b). 

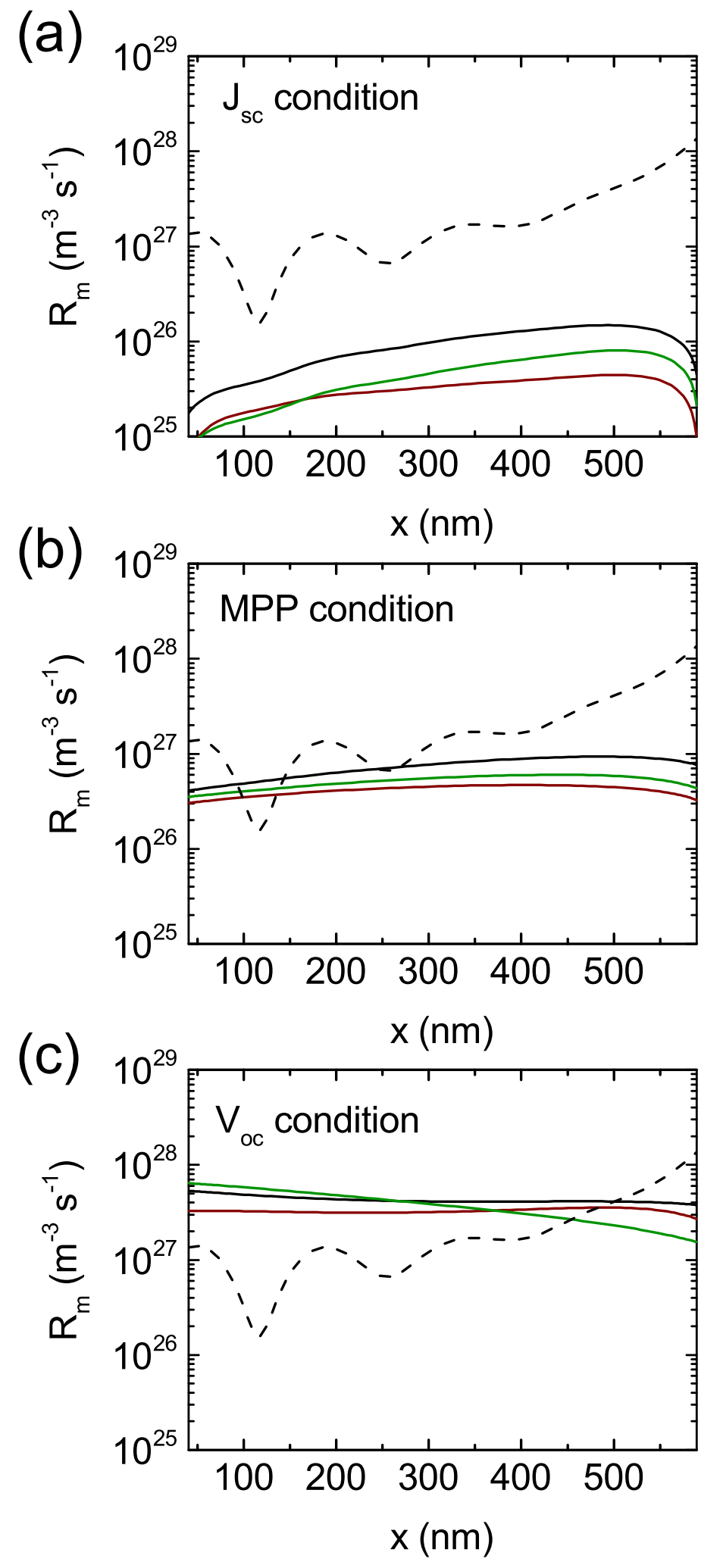

FIG. S12. Spatial simulation results for monomolecular recombination rate constant at a) short-circuit (SC), b) maximum power point (MPP), c) and open-circuit (OC) conditions at 1 sun illumination. The simulation distance is in respect to cathode $(\mathrm{Au})$ electrode. Black, red and green lines represent perovskite solar cells with $\mathrm{Pb}-\mathrm{Br}, \mathrm{FA}-\mathrm{Br}$ and $\mathrm{Cs}-\mathrm{Br}$ bromide source, respectively. The black dashed line shows the generation profile. The parameters used for simulation are shown in Table 1. 
(a)

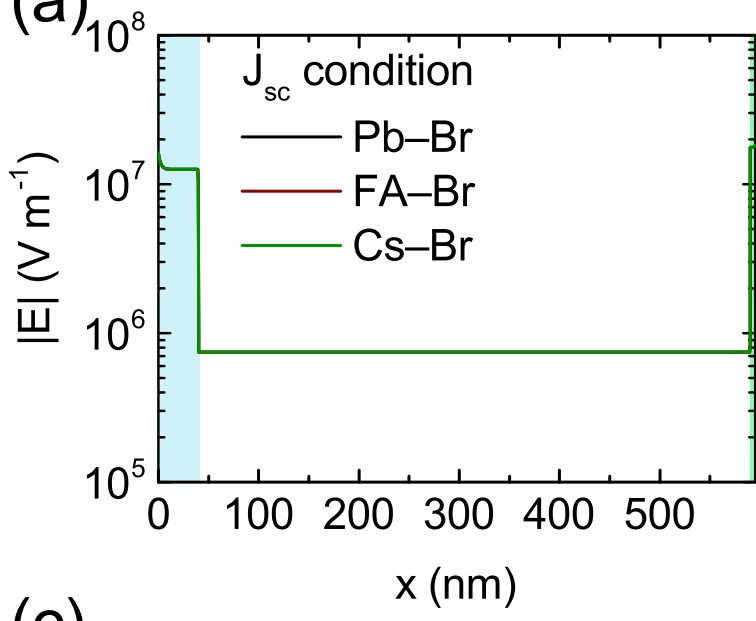

(c)

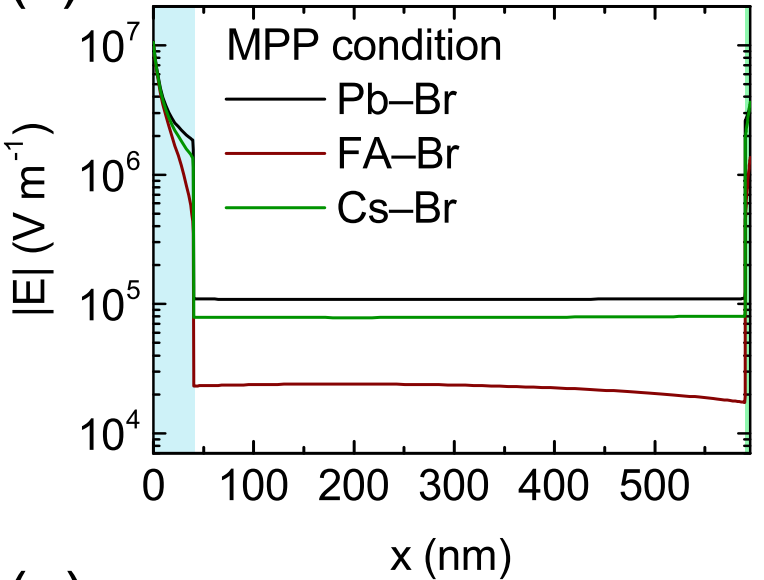

(e)

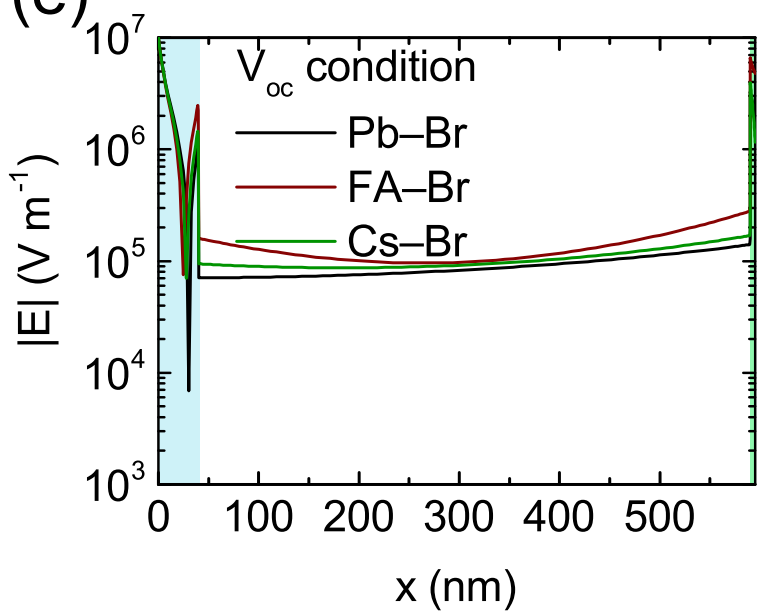

(b)

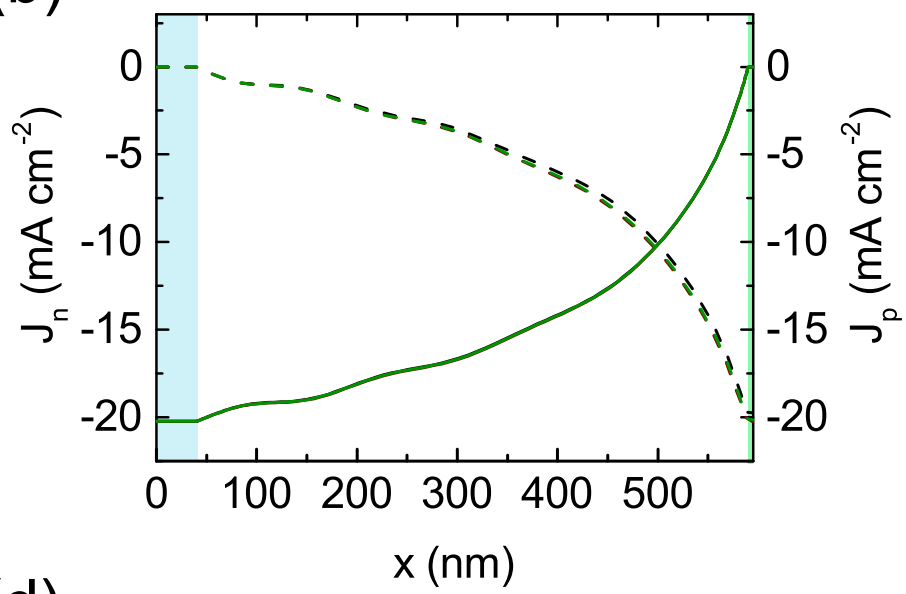

(d)

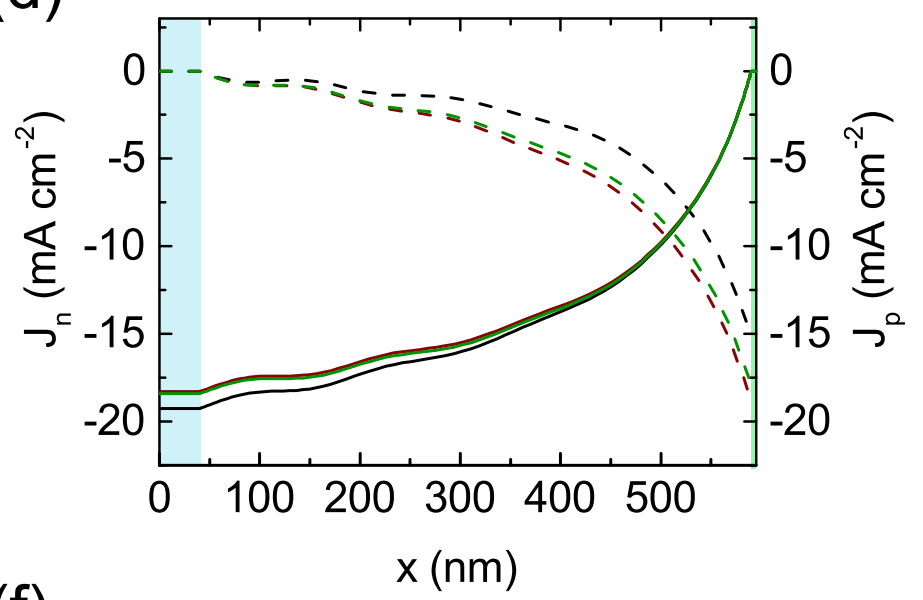

(f)

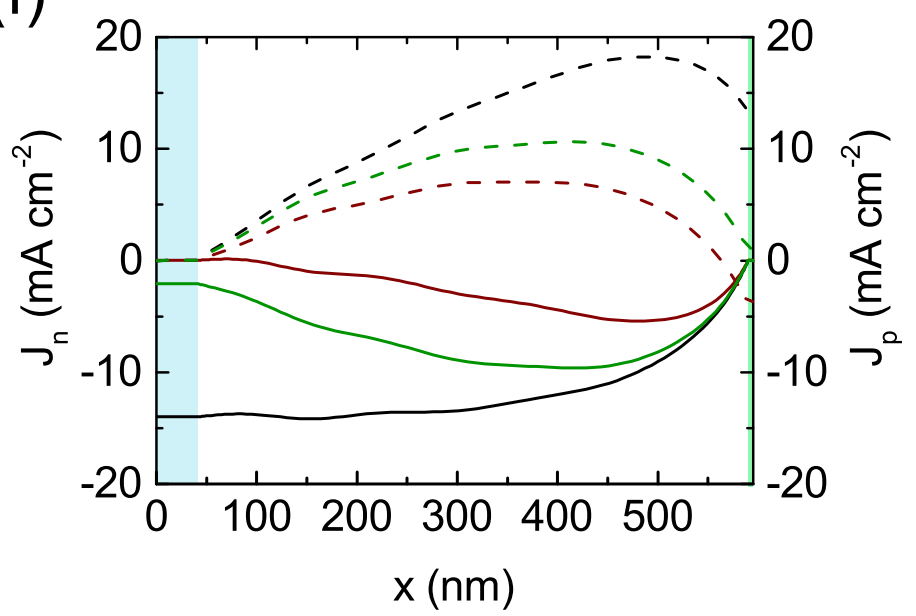

FIG. S13. Spatial simulation results for short-circuit (SC), maximum power point (MPP), and open-circuit (OC) conditions at 1 sun illumination. a,c,e) Absolute electric field distribution, b,d,f) electron (solid line) and hole (dash line) photocurrents. Black, red and green lines represent perovskite solar cells with $\mathrm{Pb}-\mathrm{Br}, \mathrm{FA}-\mathrm{Br}$ and $\mathrm{Cs}-\mathrm{Br}$ bromide source, respectively. The parameters used for simulation are shown in Table 1. 


\section{REFERENCES}

[1] Głowienka, D.; Szmytkowski, J. Numerical Modeling of Exciton Impact in Two Crystalographic Phases of the Organo-lead Halide Perovskite $\left(\mathrm{CH}_{3} \mathrm{NH}_{3} \mathrm{PbI}_{3}\right)$ Solar Cell Semicond. Sci. Technol. 2019, 34, 035018

[2] Pettersson, L.A.A.; Roman, L.S.; Inganäs, O. Modeling Photocurrent Action Spectra of Photovoltaic Devices Based on Organic Thin Films J. Appl. Phys. 1999, 86, 487-496

[3] Burkhard, G.F.; Hoke, E.T.; McGehee, M.D. Accounting for Interference, Scattering, and Electrode Absorption to Make Accurate Internal Quantum Efficiency Measurements in Organic and Other Thin Solar Cells Adv. Mater. 2010, 22, 32933297

[4] Głowienka, D.; Zhang, D.; Di Giacomo, F.; Najafi, M.; Veenstra, S.; Szmytkowski, J.; Galagan, Y. Role of Surface Recombination in Perovskite Solar Cells at the Interface of $\mathrm{HTL} / \mathrm{CH}_{3} \mathrm{NH}_{3} \mathrm{PbI}_{3}$ Nano Energy 2020, 67,104186

[5] Simmons, J.G.; Taylor, G.W. Nonequilibrium Steady-State Statistics and Associated Effects for Insulators and Semiconductors Containing an Arbitrary Distribution of Traps Phys. Rev. B 1971, 4, 502-511

[6] MacKenzie, R.C.I.; Kirchartz, T.; Dibb, G. F. A.; Nelson, J. Modeling Nongeminate Recombination in P3HT:PCBM Solar Cells J. Phys. Chem. C 2011, 115, 9806-9813

[7] Hernández-García, L.F.; Ramírez-Sánchez, O.; Cabrera-Arenas, V.; Reséndiz-Mendoza, L.M. A Gaussian Model for Recombination via Carrier-trap Distributions in Organic Solar Cells J. Comput. Electron. 2016, 15, 1103-1109

[8] Hawks, S.A.; Gang, G.; Yang, Y.; Street, R.A. Band Tail Recombination in Polymer:fullerene Organic Solar Cells J. Appl. Phys. 2014, 116, 074503

[9] Kirchartz, T.; Pieters, B.E.; Kirkpatrick, J.R.; Nelson, J. Recombination via Tail States in Polythiophene:fullerene Solar Cells Phys. Rev. B 2011, 83, 115209

[10] Nicolai, H.T.; Mandoc, M.M.; Blom, P.W.M. Electron Traps in Semiconducting Polymers: Exponential Versus Gaussian Trap Distribution Phys. Rev. B 2011, 83, 195204

[11] Lundstrom, M.S.; Schwartz, R.J.; Gray, J.L. Transport Equations for the Analysis of Heavily Doped Semiconductor Devices Solid-State Electron. 1982, 24, 195-202

[12] Lundstrom, M.S.; Schuelke, R.J. Numerical Analysis of Heterostructure Semiconductor Devices IEEE Trans. Electron. Devices 1983, 30, 1151-1159

[13] Gruber, M.; Stickler, B.A.; Trimmel, G.; Schürrer, F.; Zojer, K. Impact of Energy Alignment and Morphology on the Efficiency in Inorganic-organic Hybrid Solar Cells Org. Electron. 2010, 11, 1999-2011

[14] Scharfetter, D.L; Gummel, H.K. Large-signal Analysis of a Silicon Read Diode Oscillator IEEE Trans. Electron Devices $\mathbf{1 9 6 9}, 16,64-77$ 